This suggests that in a future theory lengths may enjoy a more fundamental status than times. This need not conflict with relativistic invariance within its valid field, that is, where four co-ordinates are used, for the new theory may take advantage of the fact that in quantum-mechanical stationary states there is no observable time co-ordinate.

43 Courtfield Road,

L. L. Whyte

London, S.W.7.

Aug. 30.

'A fuller treatment, with references on lengths, will appear in B.J. Phil. Sci., 1 .

"Whyte, L. L., Z. Phys., 56, 809 (1929); "Critique of Physics" (1931). Fürth, R., Z. Phys., 57, 429 (1929). Podolsky, B., Phys. Rev., 46

Flint, H. S., Proc. Roy. Soc., A, 159, 45 (1937)'

${ }^{3}$ Heisenberg, W', Z. Phys., 101, 533 (1936).

\section{Decay Energy of Beryllium-8 Nuclei observed in Cosmic Ray Stars}

DURING the examination of about three thousand cosmic stars in Ilford $G 5$ plates $200 \mu$ thick, exposed at balloon altitude, we observed in several cases two prongs of comparable length, making a small angle with each other, ending in the emulsion and looking like $\alpha$-particles of about $10-20 \mathrm{MeV}$.

One may suppose that such pairs are the decay products of beryllium-8 nuclei, ejected from the nucleus and disintegrating before travelling an observable distance, according to the known reaction $\mathrm{Be}^{8} \rightarrow 2 \mathrm{He}^{4}+Q$.

On this assumption, the kinetic energy $Q$ of the two $\alpha$-particles in the centre-of-mass system can be easily computed from the range of the $\alpha$-particles and the initial angle of their tracks ${ }^{1}$.

A rather narrow band of energy for the value of $Q$ was found about $100 \mathrm{keV}$. (16 pairs between 63 and $100 \mathrm{keV}$.). A broader and lower peak was also observed around $600 \mathrm{keV}$. (12 pairs between 500 and $750 \mathrm{keV}$.). Only four cases were found outside these two peaks.

In the $100-\mathrm{keV}$. band we have six events which are readily analysed, corresponding to $Q=76,94,89$, 91, 77 and $92 \mathrm{keV}$. The main cause of error is, in most cases, the difficulty in measuring the angle; this is principally due to the scattering of the tracks. The estimated error ranges from $\pm 9 \mathrm{keV}$. to $+19 \mathrm{keV}$.

In ten other events, for which the length of the tracks did not allow a definite identification of the particles, the measured values of $Q$ vary from 63 to $100 \mathrm{keV}$., with possible errors ranging from \pm 25 to +50 $\mathrm{keV}$.

The decay energy of beryllium-8 in the ground-state has already been measured by various authors, using other methods. Recent results are those of Hemmendinger ${ }^{2}$ ( $103 \pm$ $10 \mathrm{keV}$.), and Tollestrup, Fowler and Lauritsen ${ }^{3}(89 \pm 5 \mathrm{keV}$.).

Therefore, the 100-keV. peak we have observed can be attributed to beryllium-8 nuclei, emitted in the ground-state. Our measurements give a value $Q=85 \pm 10 \mathrm{keV}$. , which is in good agreement with the previous results.

The broader band around $600 \mathrm{keV}$. may indicate the existence of some other nuclear process.

Sincere thanks are due to Prof. Leprince-Ringuet for his advice and direction, to the balloon staff of the laboratory of the Ecole Polytechnique, and to Françoise Bousser and Mady Rougeul for the preliminary examination of the plates.

Laboratoire de l'École Polytechnique,

J. Crussard

Paris. July 9.

${ }^{1}$ Similar work has already been done on this subject by D. H. Perkins (private communication).

${ }^{2}$ Hemmendinger, Phys. Rev., 73, 806 (1948); 75, 1267 (1949).

${ }^{3}$ Tollestrup, Fowler and Lauritsen, Phys. Rev., 76, 428 (1949).

\section{Slow Neutron Cross-Sections of Molyb- denum and Bromine}

THE total neutron cross-sections of molybdenum and bromine have been measured between 0.05 and $10 \mathrm{eV}$. using a crystal spectrometer, and between 0.0025 and $0.1 \mathrm{eV}$. using a mechanical time-of-flight spectrometer. The crystal spectrometer is of the type described by Sturm $^{1}$ using the (110) planes of a calcium fluoride crystal in transmission. The mechanical spectrometer is similar to the one described by Brill and Lichtenberger ${ }^{2}$. Both instruments use the Harwell pile as a source of neutrons.

The molybdenum cross-section was measured using a $2 \frac{1}{2}$-in. square piece of molybdenum sheet $(15 \cdot 7 \mathrm{gm}$. per cm. ${ }^{2}$ ) which had been produced by rolling bars prepared by powder-metallurgy technique. (The sheet consisted of several thicknesses of 99.97 per cent pure molybdenum foil supplied by Johnson, Matthey and Co., Ltd.). The total cross-section as a function of energy is shown in Fig. 1. In the region from 0.0025 to 0.03 $e V$. the elastic scattering cross-section has been calculated from the theory of the transmission of slow neutrons through micro-crystalline materials, and the theoretical curve shown has been obtained by adding to these values the absorption cross-section

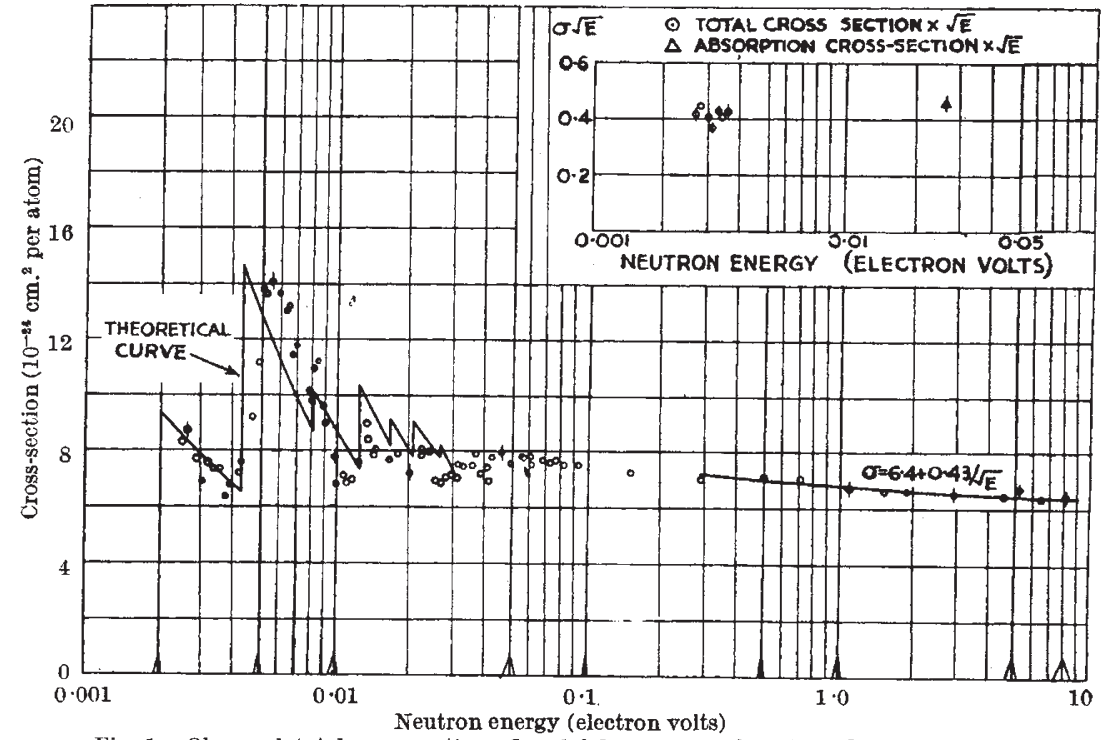

Fig. 1. Observed total cross-section of molybdenum as a function of neutron energy 